\title{
Mirroring and making sense of others
}

\section{Corrado Sinigaglia}

In a recent Review (The functional role of the parieto-frontal mirror circuit: interpretations and misinterpretations. Nature Rev. Neurosci. 11, 264-274 (2010)) $)^{1}$, Giacomo Rizzolatti and I were aiming to highlight the functional role of the parieto-frontal mirror network in understanding the actions and intentions of others. Leonhard Schilbach has recently argued (A second-person approach to other minds. Nature Rev. Neurosci. 20 May 2010 (doi: 10.1038/nrn2805-c1)) $)^{2}$ that, although "well balanced", the Review does not take into account the enactive approach to social cognition and so overlooks the fundamental difference between being directly engaged in interaction with someone else (that is, having a second-person perspective) and merely observing others (assuming a third-person perspective). This putative fault would be particularly relevant because, according to Schilbach ${ }^{2}$, our understanding of how the mirror and 'mentalizing networks' contribute to social cognition would depend on being "able to investigate real-time interactions between individuals in an ecologically valid way"2.

There is no doubt that the shift from sharing to coordinating and joining actions and intentions constitutes a crucial step in social cognition ${ }^{3,4}$. And it is true that social neuroscience needs to make use of experimental paradigms that are as ecologically plausible as possible. Indeed, most of the more intriguing properties of the mirror mechanism could be found by assuming ecological paradigms ${ }^{5,6}$. However, I think it is misleading to characterize the mirror-based action and intention understanding as a pure third-person grasp of other individuals' mental states that is performed in a mere observational - that is, detached - stance ${ }^{7}$, as it fails to appreciate the full implications of the discovery of the mirror system.

The functional properties of the parietofrontal mirror circuit indicate that our ability to act shapes how we make sense of others' behaviour to the extent that the latter can be construed as a modality of the former, at least at the basic level ${ }^{8}$. By mapping the action of others onto our own motor repertoire, the mirror mechanism not only unifies action and perception, but also undermines the sharp distinction between the observational and the engaged stance. In this regard, it is crucial to avoid confusing the experimental setting of most mirror experiments with the function of the mirror mechanism. It is true that mirror investigations usually attempted to uncouple the acting and perceiving phases, because this was the only way in which the motor responses to the observation of others' actions could be convincingly labelled as 'mirror'. But this does not signify that a mirror-based understanding of others is detached or disengaged - that is, purely spectatorial in nature. Quite the opposite: what the mirror mechanism suggests is that our making sense of others can be enactive in nature even when we are just observing others' actions. In other words, it suggests that the mere observation of others' actions can be considered a limit case of an interaction ${ }^{9}$. For this reason, we characterized mirror-based action understanding as an understanding "from the inside"; that is, as an understanding of the observed action as one's own motor possibility ${ }^{1}$.
This does not deny the relevance of attempts to investigate mirror responses in real-time social interactions. However, such investigation needs a preliminary clarification both of the basic functional properties of the parieto-frontal mirror circuit and also of the relation between the mirror-based understanding of actions and intentions and the fully fledged 'mindreading' process. As far as the latter is concerned, I cannot agree on the notion that the difference between the mentalizing network and the mirror network and their complementary roles in cognition could be reduced to "a function of direct engagement in real-time interaction"2. Of course, mirroring and mentalizing can be modulated by interactions. However, such modulation could not be detected without a prior understanding of the different aspects of action description that are involved both in the mirror mechanism and in the mentalizing processing. This prior understanding was the main aim of the Review.

Corrado Sinigaglia is at the Department of Philosophy, University of Milan, Milan, Italy.

E-mail: corrado.sinigaglia@unimi.it doi:10.1038/nrn2805-c2

1. Rizzolatti, G. $\&$ Sinigaglia, C. The functional role of the parieto-frontal mirror circuit: interpretations and misinterpretations. Nature Rev. Neurosci. 11 264-274 (2010).

2. Schilbach, L. A second-person approach to other minds. Nature Rev. Neurosci. 20 May 2010 (doi: 10.1038/nrn2805-c1).

3. Sebanz, N. Bekkering, H. \& Knoblich, G. Joint action: bodies and minds moving together. Trends Cogn. Sci. 10, 70-76 (2006).

4. Knoblich, G. \& Sebanz, N. Evolving intentions for social interaction: from entrainment to joint action. Philos. Trans. R. Soc. Lond. B Biol. Sci. 363 , 2021-2031 (2008).

5. Rizzolatti, G., Fogassi, L. $\&$ Gallese, V. Neurophysiological mechanisms underlying the understanding and imitation of action. Nature Rev. Neurosci. 2, 671-670 (2001).

6. Rizzolatti, G. \& Sinigaglia, C. Mirrors in the Brain. How our Minds Share Actions and Emotions (Oxford University Press, New York/Oxford, 2008).

7. De Jaegher, H. $\propto$ Di Paolo, E. Participatory sensemaking. An enactive approach to social cognition. Phenom. Cogn. Sci. 6, 485-507 (2007).

8. Sinigaglia, C. in Enacting Intersubjectivity: A Cognitive and Social Perspective to Study of Interactions (eds Morganti, F., Carassa, A. \& Riva, G.) 17-32 (IOS Press, Amsterdam, 2008).

9. Sinigaglia, C. Mirror in action. J. Conscious. Stud. 16, 309-334 (2009). 